\title{
Challenges in the design, conduct, analysis, and reporting in randomized clinical trial studies: A systematic review
}

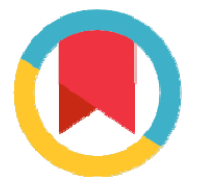

\author{
Fatemeh Varse ${ }^{1}$, Leila Janani ${ }^{2,3}$, Yousef Moradi ${ }^{4}$, Masoud Solaymani-Dodaran ${ }^{5}$, \\ Hamid Reza Baradaran ${ }^{6,7}$, Shahnaz Rimaz*1,8
}

Received: 21 Aug 2018

Published: 30 Apr 2019

\section{Abstract}

Background: Randomized clinical trials have been considered as the gold standard for evaluating the effectiveness and safety of medical interventions; however, there are major barriers to their design, conduct, analysis, and reporting. They are multidisciplinary and involve different steps and face a variety of challenges that may vary from one country to another. The aim of this study was to provide a comprehensive presentation of the challenges of clinical trial studies in different steps including design, conducting, analysis, and reporting.

Methods: In this study, all original articles conducted during 1991-2017 that reviewed the barriers to clinical trial studies at one of the steps of design, conducing, analysis, and reporting of the results in Medline (through PubMed), Embase, Web of Sciences, Scopus, and Google Scholar were considered. The searched keywords were as follow: challenges, barriers, and randomized clinical trial.

Results: The following barriers in different steps of randomized clinical trials were identified: general barriers include insufficient knowledge and understanding of clinical research and research methodology, barriers to ethical and regulatory systems, and lack of funding. The investigator-initiated trials may face similar problems to those of sponsor-initiated trials, such as handling regulatory systems, administrative and financial issues, multiple languages, and different patient compensation approaches. The challenge related to design was poor planning. Other challenges were lack of manpower and financial resources, inappropriate statistical methods for analysis (analysis challenges), and challenges related to reporting which include selective reporting.

Conclusion: Based on the results of this systematic review, the most important challenges were barriers related to handling ethical and regulatory systems, patient recruitment, and lack of budget and skilled staff for conducting clinical trials. Training to improve the quality of randomized clinical trial studies in different steps and levels was the most important recommendation in these studies.

Keywords: Barriers, Challenges, Clinical trials, Systematic review

Conflicts of Interest: None declared

Funding: Iran University of Medical Sciences

\section{*This work has been published under CC BY-NC-SA 1.0 license. \\ Copyright $($ Iran University of Medical Sciences}

Cite this article as: Varse F, Janani L, Moradi Y, Solaymani-Dodaran M, Baradaran HR, Rimaz S. Challenges in the design, conduction, analysis, and reporting randomized clinical trial studies: A systematic review. Med J Islam Repub Iran. 2019 (30 Apr);33:37. https://doi.org/10.47176/mjiri.33.37

\section{Introduction}

Randomized clinical trials (RCTs) are considered as the gold standard for evaluating the effectiveness and safety

Corresponding author: Dr Shahnaz Rimaz, rimaz.sh@iums.ac.ir

1. Student Research Committee, Iran University of Medical Sciences, Tehran, Iran

2. Preventive Medicine and Public Health Research Center, Iran University of Medical Sciences, Tehran, Iran

3. Department of Biostatistics, School of Public Health, Iran University of Medical Sciences, Tehran, Iran

4. Department of Epidemiology, School of Public Health, Iran University of Medical Sciences, Tehran, Iran

5. Minimally Invasive Surgery Research Center, Rasoul Akram Hospital, Iran University of Medical Sciences, Tehran, Iran

6. Endocrinology Research Center, Institute of Endocrinology and Metabolism, Iran University of Medical Sciences, Tehran, Iran

7. Ageing Clinical \& Experimental Research Team, Institute of Applied Health Sciences, University of Aberdeen, Aberdeen, Scotland, UK

8. Radiation Biology Research Center, Department of Epidemiology, School of Public Health, Iran University of Medical Sciences, Tehran, Iran of medical interventions (1). These types of studies are known as the basis for evidence-based decision making

$\uparrow$ What is "already known" in this topic:

Previous studies have investigated the challenges of clinical trials only in the conducting step. Most of these studies reported challenges in developing countries.

\section{$\rightarrow$ What this article adds:}

This study provided a comprehensive review of the challenges of clinical trial studies in different steps including design, conducting, analysis, and reporting in both developing and developed countries. 
and the gold standard for clinical research. In recent years, a major scientific revolution to decide on evidence-based health care has led to the development of clinical trials (2). A well-conducted randomized clinical trial can be the most reliable method for measuring the effectiveness of clinical interventions (3).

Although clinical trial studies have been considered as the gold standard, the emphasis on the quality of these studies for their effects has always been taken into account. These studies are multidisciplinary and involve different steps, so they may face a variety of challenges that vary from one country to another.

One of the basic principles for conducting a clinical trial is that the collected data should be monitored; such regular monitoring helps to ensure that the risks to the participants are not more than expected and the study is done properly (4). Many universities have less awareness of the research priorities of clinical studies (5). Conducting these studies in developing countries often causes ethical, organizational, cultural, and infrastructural barriers for researchers, pharmaceutical companies, sponsors, and regulatory agencies (2).

Due to the unique social and economic conditions of India (low literacy, poverty, lack of public medical facilities) and the unique relationships between doctors and patients in this country, patients are exposed to the most vulnerable conditions in the process of clinical trials. The main concerns are related to the process of obtaining informed consent and compensation for participants due to injury or death (6). Patient recruitment is one of the main barriers to conducting these studies. Performing a trial of all medical expertise, issues on inequality, representation, and access to health care services may raise problems in patient recruitment, but the barriers to research partnerships for minorities are through the frameworks of cultural and therapeutic misconception, poor health literacy, lack of trust in the therapeutic system, or fear of relevance of the experiments $(7,8)$. Even if the costs of randomized clinical trials are comparable to the costs of standard care, lack of insurance coverage will prevent entry or registration (6).

The randomized clinical trials (RCTs) reviewed and published in leading journals are considered as the highest level of evidence in medicine. However, this is not always the case and RCTs, even when reported in these journals, can be misleading by 2 mechanisms: (1) timeliness or poor design in RCTs; (2) misinterpretation of even welldesigned RCTs.

Several limitations may decrease the value of these studies: defects in patient selection, physician competence, randomization, applicability, endpoints, and the population being studied. Another problem with articles describing RCTs is the potential for the conclusions of the trial report to be misleading because of error or bias. This together with subsequent misinterpretation of the trial results or conclusions by others can make the effect of the trial misleading with an unintended detrimental result on medical practice (9).

Although the researcher may limit biasing through random allocation or blinding methods, clinical trial results may still be less acceptable (3).

Clinical trial studies have 4 steps of design, conducting, analysis, and reporting of results. Also, in these studies, different people are involved with different majors, which may result in some challenges associated with the conditions of each country. Therefore, the aim of this study was to provide a comprehensive review of the challenges in conducting different steps of clinical trial studies (design, conducting, analysis, and reporting) in both developing and developed countries.

\section{Methods}

This systematic review was prepared and reported based on the Preferred Reporting Items for Systematic Reviews and Meta-Analyses (PRISMA) checklist and flow diagram (10) (Fig. 1).

All original articles presenting the barriers to clinical trial studies at one of the steps of design, conducting, analysis, and reporting of the results and meeting the inclusion and exclusion criteria have been included in this review.

\section{Inclusion criteria}

- Studies that reported challenges in 4 steps of clinical trials (design, conducting, analysis, reporting)

- Studies published in English

- Studies published during 1991-2017

\section{Exclusion criteria}

- Studies that reported clinical challenges in the conducting of clinical trials in different areas of diseases.

\section{Search strategy}

The search syntax was developed based on PubMed (Medline) and included 45 related keywords and was modified based on other international databases. Databases (Medline-PubMed, Embase, Web of Sciences, Scopus, and Google Scholar) were sought for relevant scientific evidence up to October 2017. The search strategy was limited to English articles published during 19912017 (Table 1).

\section{Article screening and selection}

In the first stage, titles and abstracts of all articles were evaluated by 2 researchers (FV, YM) independently. Then, full-texts were assessed to decide on relevancy of the articles. The researchers discussed the disagreement, if any, and in case an agreement was not reached, the third author (LJ) evaluated the study. 


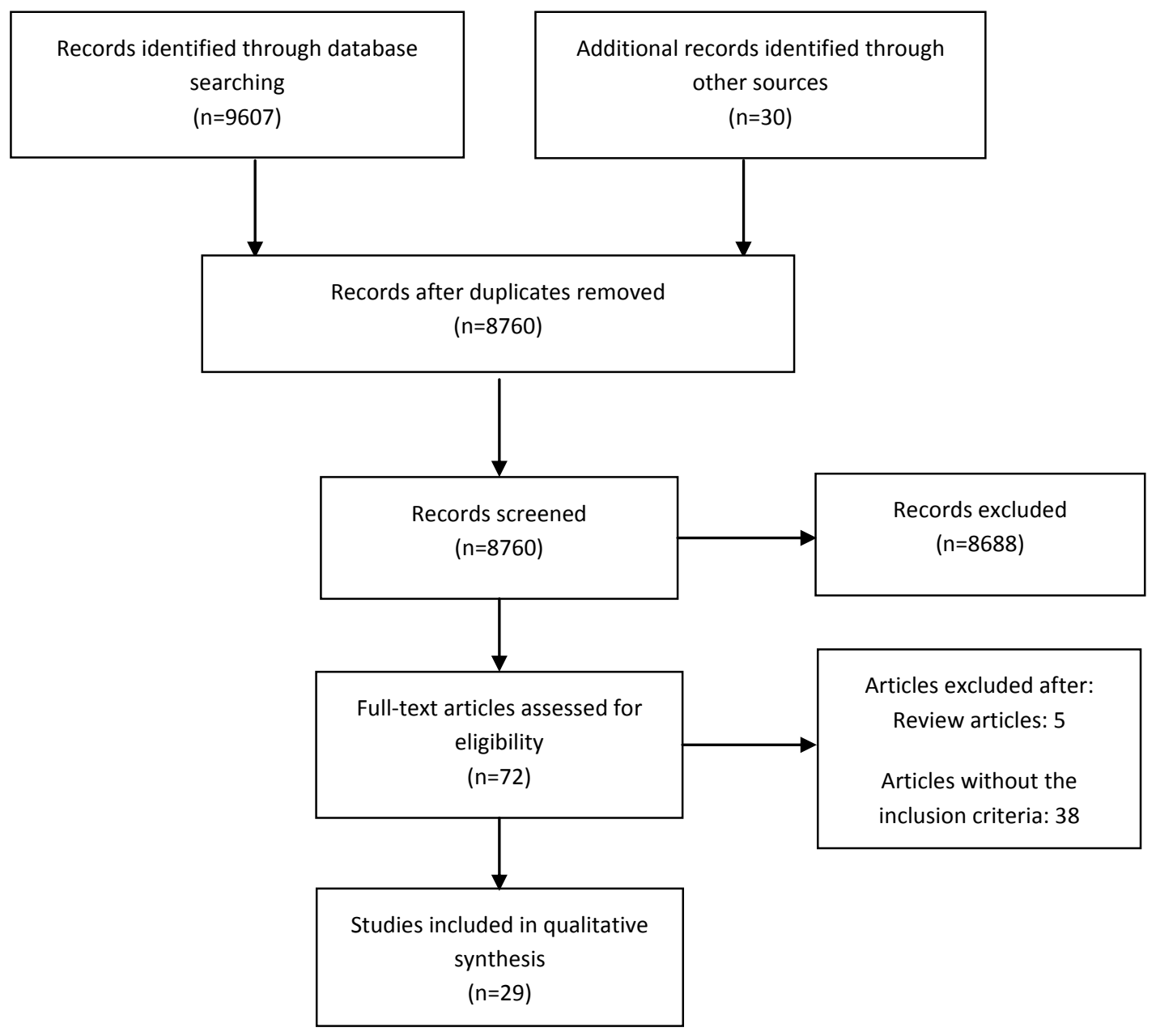

Fig. 1. PRISMA 2009 flow diagram for study selection

Table 1. Search strategy

\section{PubMed/Medline}

("clinical trial"[tiab] OR "Clinical study" [tiab] OR "Controlled clinical trial” [tiab] OR "randomized clinical trial”"[tiab] OR "randomized controlled trial” [tiab] OR "Cross Over Design" [tiab] OR "Cross Over Studies" [tiab] OR "Crossover procedure" [tiab] OR "Field Trial"[tiab] OR RCTs[tiab] OR "Community Trial" [tiab] OR "Factorial Design" [tiab] OR "Cluster randomized design" [tiab] OR "Open trial" [tiab] OR "open study" [tiab] OR "Mega trial" [tiab] OR "Intervention Studies" [tiab] OR "interventional study" [tiab] OR "intervention trial" [tiab] OR "interventional studies" [tiab] OR "Pragmatic Clinical Trial" [tiab] OR "Zelen's design" [tiab] OR "Single Arm Trials" [tiab] OR "Parallel Group Design" [tiab] OR "parallel design" [tiab] OR "clinical epidemiology" [tiab]) AND (Barriers[tiab] OR Limitations[tiab] OR Problems[tiab] OR Challenges[tiab] OR Difficulties[tiab] OR mistakes[tiab] OR setback[tiab] OR hitch[tiab] OR trouble[tiab] OR hassle[tiab] OR Defects[tiab] OR Bugs[tiab] OR flaws[tiab] OR weaknesses[tiab] OR faults[tiab] OR restriction[tiab] OR imperfection[tiab] OR wrongs[tiab] OR blunders[tiab] ) AND 1991/01/01[DP]:2017/10/20[DP])

\section{Quality Assessment}

The quality of each study was assessed according to Kuper (11). The criteria contain 6 major questions that address different key areas (design sample, data collection, analysis, reporting), each of which are answered as 'good', 'fair', and 'poor'. Based on the literature, a quality score was given for each question using the following definitions: Good: sufficient details are provided and well justified; fair: most information is given and justified but some information is missing; poor: no or few descriptive details are provided. The purpose of the quality assessment was not to establish thresholds to exclude the studies, but rather to distinguish between studies in overall contribution to the review (12).

\section{Data extraction}

All papers included in the present study were evaluated independently by 2 researchers (FV, YM). They discussed the disagreements, if any, and in case an agreement was not reached, a third author (LJ) evaluated the study. A structured checklist was used to extract the data on the (1) name of first author and title, (2) country, (3) year of publication, (4) recommendations, (5) priority populations, (6) focus, and (7) outcomes. Additional information on the study results was extracted with respect to type of instruments.

\section{Results}

In the first step, 9637 studies were evaluated, of which 877 were duplicate articles and excluded. Then, 8760 re- 
maining articles were evaluated based on their abstracts and 8688 irrelevant articles were excluded. In the next stage, full-texts were assessed and 43 articles were removed. Finally, after examining the studies and applying the inclusion and exclusion criteria, 29 studies remained and their results were evaluated. The following barriers in different steps of randomized clinical trials were identified: investigator-initiated trials may face similar difficulties with trials sponsored by international bodies. These difficulties will be related to handling of the regulatory bodies, administrative and financial bottlenecks, multiple languages, patient compensation options, interviewer compensation modalities, and additional documents required to facilitate data collection. Among the 4 stages of design, conducting, analysis, and reporting, the greatest challenges were related to conducting the randomized clinical trial. Challenges related to conducting were as follow: ethical and regulatory system obstacles $(2,5,6$, 13-19); financial bottle necks $(2,5,6,14-16,20-22)$; lack of skilled personnel $(6,14-16,18,21,22)$; barriers to patient recruitment $(5-7,23-26)$; lack of infrastructure $(5,6$, $15,16,21,22)$; lack of awareness and motivation $(6,15$, $16,18,27)$; lack of time $(5,14-16,27)$; lack of knowledge of the general population $(6,24-27)$; lack of knowledge among the research professionals $(25,26,28)$; lack of insurance coverage $(24,26)$; conflict of interest concerns $(4,25)$; lack of registry $(28,29)$; barriers to blinding $(19$, $30)$; issues in compensation calculation $(2,6)$; administrative bottle necks $(2,18)$; lack of integration between clinical trials and patient care (20); lack of research environment (5); lack of transparency (17); lack of tools (20); operational hurdle (15); and multiple languages (2). Other challenges were poor planning $(7,20)$, lack of equipoise $(25,31)$ (design challenges), non-use of appropriate sta- tistical methods for analysis (32) (analysis challenges) and challenges related to reporting which include selective reporting $(3,22)$, failure to report protocol $(27,33)$, incomplete data reporting (17), and difficulty in clinical trial report $(29,34)$ (Table 2).

\section{Discussion}

As clinical trials usually affect patient care and management more than other types of studies do, it is highly important to consider the highest standards for writing, conducting, analyzing, and reporting these studies (35). In this study, the literature on barriers facing clinical trial studies in different steps (design, conducting, analysis, reporting) were reviewed. The greatest barriers were ethical and regulatory system obstacles, financial bottlenecks, lack of skilled personnel, and barriers to patient recruitment. In addition, several other challenges emerged from the research literature: lack of infrastructure, lack of awareness and motivation, lack of time, lack of knowledge among the general public, and lack of knowledge among the research professionals. By contrast, an article discussed the opportunities and challenges in conducting clinical trials globally. Informed consent, data quality, differences in medical practice, acceptability of foreign data, and ethical factors were global clinical trials challenges (36). A systematic review related to barriers for conducting clinical trials in developing countries indicated that the greatest challenge that researchers faced in developing countries was lack of financial and human capacity (37). However, a systematic review on physician barriers in RCTs for cancer and other illnesses in the developed world identified lack of time as a major barrier (38). Another study discussed barriers to the conduct of randomized clinical trials within all disease areas. In that

Table 2. Barriers for designing, conducting, analyzing, and publishing clinical trials

Barriers for designing, conducting, analyzing, and publishing clinical trials

Thematic barriers
Barriers for designing a clinical trial

Barriers for conducting a clinical trial

Barriers for analyzing a clinical trial Barriers for publishing a clinical trial

\begin{tabular}{|c|c|}
\hline Subthemes & Reference \\
\hline Poor design & $(7,20)$ \\
\hline Lack of equipoise & $(25,31)$ \\
\hline Financial bottlenecks & $(2,5,6,14-16,20-22)$ \\
\hline Lack of insurance coverage & $(24,26)$ \\
\hline Lack of knowledge among the research professionals & $(25,26,28)$ \\
\hline Lack of knowledge among general public & $(6,24-27)$ \\
\hline Ethical and regulatory system obstacles & $(2,5,6,13-19)$ \\
\hline Lack of skilled personnel & $(6,14-16,18,21,22)$ \\
\hline Lack of research environment & $(5)$ \\
\hline Lack of integration between clinical trials and patient care & (20) \\
\hline Issues in compensation & $(2,6)$ \\
\hline Lack of awareness and motivation & $(6,15,16,18,27)$ \\
\hline Lack of tools & $(20)$ \\
\hline transparency Lack of & (17) \\
\hline Lack of infrastructure & $(5,6,15,16,21,22)$ \\
\hline Barriers to blinding & $(19,30)$ \\
\hline Operational hurdles & $(15)$ \\
\hline Conflict of interest concerns & $(4,25)$ \\
\hline Lack of registry & $(28,29)$ \\
\hline Barriers to patient recruitment & $(5-7,23-26)$ \\
\hline Multiple languages & (2) \\
\hline Administrative bottlenecks & $(2,18)$ \\
\hline Lack of time & $(5,14-16,27)$ \\
\hline Lack of using appropriate statistical methods & $(32)$ \\
\hline Selective reporting & $(3,22)$ \\
\hline Incomplete reporting of data & $(17)$ \\
\hline Difficulty in clinical trial report & $(29,34)$ \\
\hline Failure to report protocol & $(27,33)$ \\
\hline
\end{tabular}


study, inadequate knowledge of clinical research and trial methodology, lack of funding, excessive monitoring, restrictive privacy law, lack of transparency, complex regulatory requirements, and inadequate infrastructures were barriers to randomized clinical trials (39).

Ethical and regulatory barriers were the most commonly cited reasons reported in the included studies. Developed countries perform a number of clinical trials in developing countries. In developing countries, such as India, these trials indicated that most Indians do not have access to good quality and affordable care and therefore may accept offers that might provide better quality and free treatment. They were conducted on people who were vulnerable because they could not afford good quality treatment or the most effective drugs (6). On the other hand, medications that are experimented in clinical trials in developed countries may not be used by people in developing countries due to their high cost. This subject can be an ethical problem because there is a principle in clinical trials that says the benefits of clinical trials must be more than their disadvantages and people should benefit from the result of these studies.

Another challenge was financial bottlenecks, which is reflective of the 10/90 gap in which less than $10 \%$ of health research funds in the world are directed toward problems that affect $90 \%$ of the world's population, and an even smaller percentage goes to fund researchers and health problems of developing countries $(6,40,41)$. Funding for clinical trials in developing countries comes mostly from Western countries and the pharmaceutical companies based there (42). In most low-income countries, research is a luxury because of economic constraints (43). Scarce resources in developing countries are nearly all spent on program implementation, and allocating funds for research is almost out of the equation in most development plans. Contrary to existing beliefs and practices, the lack of resources in low- and middleincome countries paradoxically increases the need for reliable health care evidence to prioritize the use of these scarce resources (44).

Conducting clinical trials is essential for evaluating therapeutic interventions, but at the same time, taking care of patients is also of crucial importance. To address this necessity, numerous international statements and guidelines on protection of human subjects have been drafted and implemented. However, the results of a study conducted in India showed that in clinical research activity, because of the unique socioeconomic conditions in India (low literacy, poverty, general lack of medical facilities) and the unique doctor-patient relationship, the patient ends up being the most vulnerable in the clinical trial process. The culture and socioeconomic conditions of any country and region may affect the quality and the stages of conducting a clinical trial. The regulatory guidelines are part of a dynamic process and will continue evolving based on the changing requirements and demands of the system. Therefore, these regulations must be carried forward by proactively responding to such challenges with suitable clarifications and/or amendments without compromising the well-being of trial participants (6).
Conducting clinical trials and obtaining informed consent in any country are subject to the cultural conditions of that country. For example, the results of a study conducted in sub-Saharan Africa showed that the collection of blood samples from individuals in clinical trial projects could be challenging and may be one of the considerable reasons for local rumors of "blood theft" or "blood sale". Such attitudes and cultures could potentially have a profound effect on the success of research projects (45). Participants' knowledge is also highly important in properly implementing and collecting data of a clinical trial. To confirm these results, a study conducted in the United States showed that at least $40 \%$ of Americans did not understand the idea of a clinical trial.

For various reasons, knowledge can be a major factor in participation. First, low knowledge or confusion about the key facts of trial methods (random allocation, informed consent, standard treatment) can disrupt participation (46). Secondly, patients are often reluctant to participate in something about which they have little knowledge (47).

The lack of knowledge is not limited to patients. One of the most frequent probable reasons for the lack of reporting was lack of awareness and shortage of knowledge about clinical trials $(48,49)$. Prior to the conduction of trials, a protocol is conducted to specify the exact steps of the task. Due to the unpredictable nature of interventions in health sciences, some changes may occur during the implementation of the trials with respect to the approved protocols. Therefore, researchers need to include these changes in their reports (50). However, excessive defects of a protocol may result in patient damage and occurrence of errors in clinical trial outcomes. Although the CONSORT statement provides a comprehensive guide to protocol defect reports, the reports of studies are not complete. Hence, as the first step to improve reporting, authors are recommended to report protocol defects based on the CONSORT statement.

The results of the studies indicated that most researchers lacked knowledge and awareness even in the early definitions of the principles of a trial. For example, a study by Schulz et al showed that most researchers failed to recognize blinding and allocation concealment, and this suggested their lack of knowledge (51).

It is recommended that this issue be continually taught to researchers, as lack of knowledge may affect all stages of clinical trials and their outcomes. A study by White et al showed that knowledge rather than attitude is the leading factor impeding registration. Investigators were very unfamiliar with international registration tools other than clinicaltrials.gov (28).

Registration of clinical trials may prevent publication bias and selective reporting, as the bias caused by selective reporting can affect meta-analyses work on the results of systematic reviews and evidence-based studies, leading to giving incorrect answers to important clinical questions. Patients, doctors, and health policymakers rely on this information in their evidence-based decision making (3). Thus, according to $\mathrm{WHO}$, registration of a clinical trial is "a scientific, ethical and moral responsibility" (52). 
It is, therefore, highly important to promote the registration of clinical trials as part of the national research policy. However, a study conducted in Argentina showed that the current percentages of internationally registered trials and participants' responses were insufficient to achieve the desired goals (28). It is important that all registrations, including integrated information elements, be electronically and widely searchable (53).

It should always be taken into consideration that clinical trial management is a public responsibility and requires a deep understanding of statistical methods.

This systematic review had limitations and strengths. It provided a comprehensive presentation of the challenges of clinical trial studies in different steps including design, conducting, analysis, and reporting, in both developing and developed countries. Because of the limited number of articles in the review, the study did not examine the similarities and differences between barriers among the different countries. Most of the included studies were qualitative. Those articles not written in English were excluded, as because the cost of translation was not feasible.

\section{Conclusion}

Among the 4 stages of design, conducting, analysis, and reporting, the most challenges were related to conduct of randomized clinical trial. The main barriers identified in the present systematic review were as follow: barriers related to handling ethical and regulatory systems, patient recruitment, lack of budget and skilled staff, lack of infrastructure, lack of knowledge among the general public, lack of awareness and motivation, and lack of time for conducting clinical trials. Barriers may vary widely depending on the context in which the clinical trials are conducted. There are several intercountry differences in culture, socioeconomic, and political contexts. Thus, findings of this review may not reflect the situation in any specific country. Providing training in different steps and levels to improve the quality of randomized clinical trial studies was the most important recommendation in these studies.

\section{Acknowledgments}

This study was funded by Iran University of Medical Sciences (grant number 97-01-27-33259).

\section{Conflict of Interests}

The authors declare that they have no competing interests.

\section{References}

1.London L, Hurtado-de-Mendoza A, Song M, Nagirimadugu A, Luta G, Sheppard VB. Motivators and barriers to Latinas' participation in clinical trials: the role of contextual factors. Contemporary clinical trials. 2015;40:74-80.

2. Mbuagbaw L, Thabane L, Ongolo-Zogo P, Lang T. The challenges and opportunities of conducting a clinical trial in a low resource setting: the case of the Cameroon mobile phone SMS (CAMPS) trial, an investigator initiated trial. Trials. 2011;12(1):145.

3. McKenzie JE, Herbison GP, Roth P, Paul C. Obstacles to researching the researchers: A case study of the ethical challenges of undertaking methodological research investigating the reporting of randomised controlled trials. Trials. 2010;11(1):28.
4. Ellenberg SS. Protecting clinical trial participants and protecting data integrity: are we meeting the challenges? PLoS Medicine. 2012;9(6):e1001234.

5.Hummers-Pradier E, Bleidorn J, Schmiemann G, Joos S, Becker A, Altiner A, et al. General practice-based clinical trials in Germany-a problem analysis. Trials. 2012;13(1):205.

6. Gupta YK, Pradhan AK, Goyal A, Mohan P. Compensation for clinical trial-related injury and death in India: Challenges and the way forward. Drug safety. 2014;37(12):995-1002.

7. Spaar A, Frey M, Turk A, Karrer W, Puhan MA. Recruitment barriers in a randomized controlled trial from the physicians' perspective-A postal survey. BMC Medical Research Methodology. 2009;9(1):14.

8. Borno H, Siegel A, Ryan C. The problem of representativeness of clinical trial participants: understanding the role of hidden costs. SAGE Publications Sage UK: London, England; 2016.

9. Veith FJ. How can good randomized controlled trials in leading journals be so misinterpreted? Journal of Vascular Surgery. 2013;57(2):3S-7S.

10. Moher D, Liberati A, Tetzlaff J, Altman DG, Group P. Reprintpreferred reporting items for systematic reviews and meta-analyses: the PRISMA statement. Physical Therapy. 2009;89(9):873-80.

11. Kuper A, Lingard L, Levinson W. Critically appraising qualitative research. BMJ. 2008;337:a1035-a.

12. Lorenc T, Petticrew M, Whitehead M, Neary D, Clayton S, Wright $\mathrm{K}$, et al. Quality assessment for the systematic review of qualitative evidence. 2014

13. Osrin D, Azad K, Fernandez A, Manandhar DS, Mwansambo CW, Tripathy $\mathrm{P}$, et al. Ethical challenges in cluster randomized controlled trials: experiences from public health interventions in Africa and Asia. Bull World Health Organ. 2009;87(10):772-9.

14. Siegfried N, Volmink J, Dhansay A. Does South Africa need a national clinical trials support unit? SAMJ. 2010;100(8):521-4.

15. Franzen SR, Chandler C, Enquselassie F, Siribaddana S, Atashili J, Angus B, et al. Understanding the investigators: a qualitative study investigating the barriers and enablers to the implementation of local investigator-initiated clinical trials in Ethiopia. BMJ open. 2013;3(11):e003616.

16. Franzen SR, Chandler C, Atashili J, Angus B, Lang T. Barriers and enablers of locally led clinical trials in Ethiopia and Cameroon: a prospective, qualitative study. The Lancet. 2013;382:14.

17. Koenig F, Slattery J, Groves T, Lang T, Benjamini Y, Day S, et al. Sharing clinical trial data on patient level: opportunities and challenges. Biom J. 2015;57(1):8-26.

18. Sulthan N. Perception of clinical research among clinical investigators in Saudi Arabia. Asian J Pharm Clin Res. 2015;8:243-6.

19. di Ruffano LF, Deeks J. Barriers to blinding: an analysis of the feasibility of blinding in test-treatment RCTS. Trials. 2015;16(S2):O30.

20. Kukafka R, Allegrante JP, Khan S, Bigger JT, Johnson SB. Understanding facilitators and barriers to reengineering the clinical research enterprise in community-based practice settings. Contemporary Clinical Trials. 2013;36(1):166-74.

21. Cardoso A, Breugelmans G, Manville C, Chataway J, Cochrane G, Snodgrass J. Africa mapping: current state of health research on poverty-related and neglected infectious diseases in Sub-Saharan Africa.[Internet]. 2014 [cited 2015 Jun 28].

22. Ioannidis JP, Caplan AL, Dal-Ré R. Outcome reporting bias in clinical trials: why monitoring matters. BMJ. 2017;356.

23. Buis LR, Janney AW, Hess ML, Culver SA, Richardson CR. Barriers encountered during enrollment in an internet-mediated randomized controlled trial. Trials. 2009;10(1):76.

24. Kim SH, Tanner A, Friedman DB, Foster C, Bergeron CD. Barriers to clinical trial participation: a comparison of rural and urban communities in South Carolina. Journal of Community Health. 2014;39(3):562-71.

25. Donovan JL, Paramasivan S, de Salis I, Toerien M. Clear obstacles and hidden challenges: understanding recruiter perspectives in six pragmatic randomised controlled trials. Trials. 2014;15(1):5.

26. Tanner A, Kim SH, Friedman DB, Foster C, Bergeron CD. Barriers to medical research participation as perceived by clinical trial investigators: communicating with rural and African American communities. Journal of Health Communication. 2015;20(1):88-96.

27. Chan AW, Hróbjartsson A. Promoting public access to clinical trial protocols: challenges and recommendations. Trials. 2018;19(1):116.

28. White L, Ortiz Z, Cuervo LG, Reveiz L. Clinical trial regulation in 
Argentina: overview and analysis of regulatory framework, use of existing tools, and researchers' perspectives to identify potential barriers. Revista Panamericana de Salud Pública. 2011;30:445-52.

29. Walker KF, Stevenson G, Thornton JG. Discrepancies between registration and publication of randomised controlled trials: an observational study. JRSM Open. 2014;5(5):2042533313517688.

30. Pocock SJ, Clayton TC, Stone GW. Challenging issues in clinical trial design: part 4 of a 4-part series on statistics for clinical trials. J Am Coll Cardiol.2015;66(25):2886-98.

31. Deng C, Hanna K, Bril V, Dalakas MC, Donofrio P, van Doorn PA, et al. Challenges of clinical trial design when there is lack of clinical equipoise: use of a response-conditional crossover design. Journal of Neurology. 2012;259(2):348-52.

32. Howard KI, Krause MS, Vessey JT. Analysis of clinical trial data: The problem of outcome overlap. Psychotherapy: Theory, Research, Practice, Training. 1994;31(2):302.

33. Sweetman EA, Doig GS. Failure to report protocol violations in clinical trials: a threat to internal validity? Trials. 2011;12(1):214.

34. Cole GD, Shun-Shin MJ, Nowbar AN, Buell KG, Al-Mayahi F, Zargaran D, et al. Difficulty in detecting discrepancies in a clinical trial report: 260-reader evaluation. Int J Epidemiol. 2015;44(3):862-9.

35. Hutton J, Williamson PR. Bias in meta-analysis due to outcome variable selection within studies. J Royal Stat Soc. 2000;49(3):35970 .

36. Bansal N. The opportunities and challenges in conducting clinical trials globally. Clinical Research and Regulatory Affairs. 2012;29(1):9-14.

37. Alemayehu C, Mitchell G, Nikles J. Barriers for conducting clinical trials in developing countries-a systematic review. International Journal for Equity in Health. 2018;17(1):37.

38. Ross S, Grant A, Counsell C, Gillespie W, Russell I, Prescott R. Barriers to participation in randomised controlled trials: a systematic review. J Clin Epidemiol. 1999;52(12):1143-56.

39. Djurisic S, Rath A, Gaber S, Garattini S, Bertele V, Ngwabyt SN, et al. Barriers to the conduct of randomised clinical trials within all disease areas. Trials. 2017;18(1):360.

40. Moon S, Bermudez J, Hoen Et. Innovation and access to medicines for neglected populations: could a treaty address a broken pharmaceutical R\&D system? PLoS Medicine. 2012;9(5):e1001218.

41. Davey S. The 10/90 report on health research 2003-2004: Global Forum for Health Research; 2004.

42. Gaillard J. Measuring research and development in developing countries: main characteristics and implications for the Frascati manual. Science, Technology and Society. 2010;15(1):77-111.

43. Harris E. Building scientific capacity in developing countries: Simply transferring knowledge and instrumentation is not enough to help developing countries build their own research base. Such efforts must be tied to national and local needs to create trust and services for society in the long term. EMBO Reports. 2004;5(1):7-11.

44. McMichael C, Waters E, Volmink J. Evidence-based public health: what does it offer developing countries? Journal of Public Health. 2005;27(2):215-21.

45. Grietens KP, Ribera JM, Erhart A, D'Alessandro U, editors. Doctors and Vampires in Sub-Saharan Africa: Ethical Challenges in Clinical Trial Research. Tropical Medicine \& International Health; 2013: Wiley-Blackwell 111 River St, Hoboken 07030-5774, NJ USA.

46. Frank G. Current challenges in clinical trial patient recruitment and enrollment. SoCRA Source. 2004;2(February):30-8.

47. Jones JM, Nyhof-Young J, Moric J, Friedman A, Wells W, Catton P. Identifying motivations and barriers to patient participation in clinical trials. Journal of Cancer Education. 2007;21(4):237-42.

48. Kao LS, Tyson JE, Blakely ML, Lally KP. Clinical research methodology I: introduction to randomized trials. Journal of the American College of Surgeons. 2008;206(2):361-9.

49. Stommel M, Wills C. Clinical research: Concepts and principles for advanced practice nurses: Lippincott Williams \& Wilkins; 2004.

50. Moher D, Hopewell S, Schulz KF, Montori V, Gøtzsche PC, Devereaux P, et al. CONSORT 2010 explanation and elaboration: updated guidelines for reporting parallel group randomised trials. BMJ. 2010;340:c869.

51. Schulz KF, Grimes DA. Blinding in randomised trials: hiding who got what. The Lancet. 2002;359(9307):696-700.

52. Organization WH. International Clinical Trials Registry Platform (ICTRP) 2012. 2012.

53. Kelsall D. Stand and declare: Opportunity and challenge of clinical trial registration. Can Fam Physician. 2006;52(10):1189. 